\title{
SEPIA - a new single pixel receiver at the APEX telescope (Corrigendum)
}

V. Belitsky ${ }^{1,2}$, I. Lapkin ${ }^{1,2}$, M. Fredrixon ${ }^{1,2}$, D. Meledin ${ }^{1,2}$, E. Sundin ${ }^{1,2}$, B. Billade ${ }^{2}$, S.-E. Ferm ${ }^{1,2}$ A. Pavolotsky ${ }^{1,2}$, H. Rashid ${ }^{1}$, M. Strandberg ${ }^{1,2}$, V. Desmaris ${ }^{1}$, A. Ermakov ${ }^{1}$, S. Krause ${ }^{1}$, M. Olberg ${ }^{2}$, P. Aghdam ${ }^{1,2}$, S. Shafiee ${ }^{1,2}$, P. Bergman ${ }^{2}$, E. De Beck², H. Olofsson², J. Conway², C. De Breuck ${ }^{3}$, K. Immer ${ }^{3}$, P. Yagoubov ${ }^{3}$, F. M. Montenegro-Montes ${ }^{4}$, K. Torstensson ${ }^{4}$, J.-P. Pérez-Beaupuits ${ }^{4}$, T. Klein ${ }^{4}$, W. Boland ${ }^{5}$, A. M. Baryshev ${ }^{5}$, R. Hesper ${ }^{5}$, J. Barkhof ${ }^{5}$, J. Adema ${ }^{5}$, M. E. Bekema ${ }^{5}$, and A. Koops ${ }^{5}$

${ }^{1}$ Group for Advanced Receiver Development (GARD), Department of Space, Earth and Environment, Chalmers University of Technology, 41296 Gothenburg, Sweden e-mail: victor.belitsky@chalmers.se

2 Department of Space, Earth and Environment, Chalmers University of Technology, Onsala Space Observatory (OSO), 43992 Onsala, Sweden

3 European Southern Observatory (ESO), Karl-Schwarzschild-Str. 2, 85748 Garching bei München, Germany

${ }^{4}$ European Southern Observatory, Alonso de Córdova 3107, Vitacura, Casilla 19001, Santiago de Chile, Chile

5 Netherlands Research School for Astronomy (NOVA), Kapteyn Astronomical Institute, Landleven 12, 9747 AD Groningen, The Netherlands

A\&A, 612, A23 (2018) https://doi.org/10.1051/0004-6361/201731458

Key words. instrumentation: detectors - techniques: spectroscopic - errata, addenda

A mistake occurred in the first paragraph of Sect. 2.1 during the production process. One should read the following sentences.

One of the major challenges of bringing the SEPIA receiver with installed ALMA cartridges to the APEX telescope is the necessity to implement tertiary optics, which should not only provide the required and frequency independent illumination of the secondary but also be compatible with the existing optical layout of the APEX Cabin A where all single-pixel heterodyne receivers are installed. Another serious constraint is a clearance of the APEX Cabin A Nasmyth tube, whose rim is limited by the elevation encoder down to $150 \mathrm{~mm}$ in diameter requiring precision alignment possibilities to avoid Band 5 receiver beam truncation. ALMA receiver cartridges have built-in cold optics optimized for their respective position inside the ALMA FrontEnd $(\mathrm{FE})$ receiver cryostat place at the antenna focal plane. In particular, depending on the cartridge position offset from the FE center, the beam tilt offset compensating is different for, for example, ALMA Band 5 - 2.38 degree and for ALMA Band 9 cartridges - 0.93 degree. SEPIA tertiary optics shall accommodate all these constrains and differences with minimum number of reflecting surfaces (thus minimizing the reflecting loss) and fit a very confined volume within the APEX Cabin A. Specific for SEPIA and in contrast to ALMA FE, we use a rotating cartridgeselection mirror. Such an optical switch addresses limitations of the Nasmyth layout when one receiver channel has access to the sky at a time (Fig. 1). The cartridge-selection mirror (NMF3, Fig. 1) with its precision computer-controlled rotating mechanism facilitates the accommodation of different ALMA cartridges having specific differences in the incoming beam positioning as outlined above. 